\title{
Experimental Verification of Digital Thermo-Optic Switches with Silicon-on-Insulator Waveguide Corner Mirror
}

\author{
Tiancheng $\mathrm{Li}^{1}$, Yunjie Shi ${ }^{1}, \mathrm{Na} \mathrm{Sun}^{1}$, Hongpeng Shang ${ }^{1}$ and D. G. Sun ${ }^{1,2, *}$ \\ ${ }^{1}$ School of Science, Changchun University of Science and Technology, 7089 Weixing Road, Changchun, JL 130022 China \\ ${ }^{2}$ School of Science, University of Ottawa, 25 Templeton Street, Ottawa, ON, K1N 6N5, Canada \\ *Corresponding author
}

\begin{abstract}
In this work, an effective Goos-Hänchen (GH) spatial shift of reflective guided-mode is implemented with a waveguide corner mirror structure and further with an efficient combination of the GH spatial shift and the thermo-optic (TO) refractive index modulation (RIM), the function of a digital TO switch is systematically simulated with the theoretical model and the professional software of finite difference time-domain (FDTD). Therefore, on the silicon-on-insulator (SOI) platform, the functions of a $1 \times 3 / 1 \times 2$ digital optical switch are experimentally demonstrated.
\end{abstract}

Keywords-digital optical switch; thermo-optic effect; Goos-Hänchen effect

\section{INTRODUCTION}

With the increasing capacity and bandwidth of optical communication network, the new configurations and technologies together of communication components and modules continue to emerge, leading to the new theoretical models and functions. Based on the planar lightwave circuit (PLC) and photonic integrated circuit (PIC), some optical functional components are playing the key roles, for example optical switch (OS), wavelength division multiplexing (WDM) device and optical splitter, etc. [1], [2] . Silicon-on-insulator (SOI) platform has presented its exclusive merits in developing high-performance functional components and systems, especially the silicon waveguide based thermo-optic (TO) switches have been attracting intensive interests [3]-[8]. In addition, the large-scale optical switching systems specially need the highly integrated optical high port-count matrix switches (HPC-MS), for which the digital optical switches (DOS) present the exclusive advancements in the performance-price effect since the output signal of a DOS is insensitive to a deviation from the modulating signal and has a high tolerance in design/fabrication, resulting in the stable switching operations.

At the begin of this century, a lot of regimes of waveguide couplers were activated for the applications of DOS systems, the first typical establishments are based on adiabatic couplers, including the $\mathrm{LiNbO}_{3}$ waveguide adiabatic coupler based electro-optic (EO) DOS by Krahenbuhl et al. in 2002 [9]. Since the silicon film of SOI-platform was reported to have the semiconductor free-carrier dispersion (FCD) effect, the high-bandwidth nanoscale waveguide EO switches have been extensively studied, an illustrative pioneer work in DOS was reported in 2009 by Campenhout et al. of the Watson Research Center at IBM based on the asymmetric cascaded configuration of Mach-Zehnder interferometer (MZI) structures. [3] In 2013, Our research group first theoretically proved the quantum Goos-Hänchen $(\mathrm{GH})$ spatial shift, and then proposed a possible regime of digital optical switches. [10]

\section{DEVICE CONCEPTS AND ThEORETICAL INVESTIGATION}

\section{A. Device Concepts and Theoretical Models}

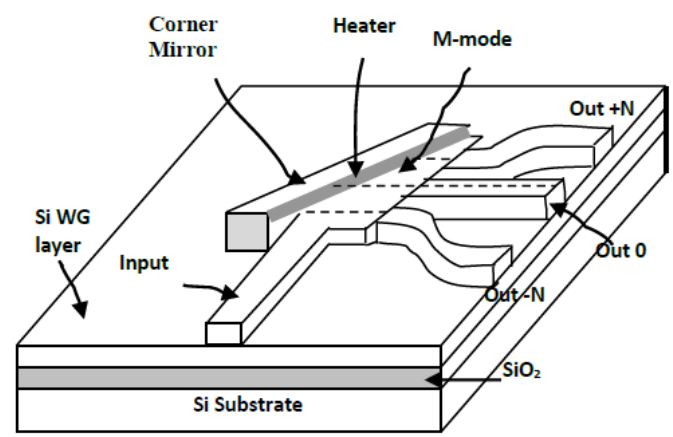

FIGURE I. SCHEMATIC CONFIGURATION OF THE SOI WAVEGUIDE CORNER STRUCTURE AND THE 1 X3 OPTICAL SWITCH BASED ON GH SHIFT WITH MICROSCALE WAVEGUIDES AND TO-RIM

Figure 1 depicts the schematic configuration of a $1 \times 3$ scale TO DOS based on SOI platform and it consists of one single-mode input waveguide, three single-mode output waveguides, a waveguide corner mirror (WCM) , an MMI structure and a heater, where the corner mirror material is $\mathrm{SiO}_{2}$. Then, the definition of GH total shift is the spatial shift $\Delta$ and the angular shift $\Theta$ produces an algebra sum through an MMI reflective waveguide structure as [10]:

$$
S_{T}=\left(\Delta+l_{m m} \Theta\right) / \cos \theta
$$

where $l_{m m}$ is the length of reflective MMI structure and $\theta$ is the incident angle. Then, with the reflection coefficient $r=R \cdot \exp (i \phi)$ and the propagation constant of the input guided-mode $k_{i n}$, the GH spatial shift and GH angular shift can respectively defined as 


$$
\begin{gathered}
\Delta=\frac{1}{k_{i n}} \cdot \frac{\partial \phi}{\partial \theta}=\operatorname{Im}(\ln r) \\
\Theta=\frac{2}{\left(k_{i n}^{2} \cdot w_{o}^{2}\right)} \cdot \frac{1}{R} \cdot \frac{\partial \phi}{\partial \theta}=\operatorname{Re}(\ln r)
\end{gathered}
$$

where $w_{o}$ is the waist of a reflected mode of approximated Gaussian beam . Our simulations show that the TE-mode can work well with this corner mirror structure, but the TM-mode cannot, so we only study this $1 \times 3 \mathrm{WCM}$ based TO switch at the TE-mode state in this work, so the amplitude reflection coefficients RTE is defined by [11]:

$$
R_{T E}=\frac{\cos (\theta)-\sqrt{\eta^{2}-\sin (\theta)}}{\cos (\theta)+\sqrt{\eta^{2}-\sin (\theta)}}
$$

where $\eta=N_{\text {eff }} / n_{m}$ is the conversion parameter between TE and TM modes if $N_{\text {eff }}$ and $n_{m}$ are the effective refractive index of the guide-mode of input waveguide and the refractive index of corner mirror material, respectively. In Ref. [10], for the eigenstate of a reflected mode during a GH spatial shift, the partial derivative meets $\partial \phi / \partial \theta=0$, so the incident angle $\theta$ should correspond to an eigenvalue of wave function $\phi(x, z)$. an important finding from Eq.(2b) is that the GH angular shift $\Theta$ pertains to the $N_{\text {eff }}$ that is determined by the input waveguide material. We know that all the guided modes of an MMI waveguide structure are the eigenstates of quantum physical process and the effective indices are the eigenvalues of all the refractive indices of optical beam at the phase velocities [10]. So, if the input waveguide channel is a single-mode, $N_{\text {eff }}$ would be only the eigenvalue determining one of the $\mathrm{GH}$ angular shifts. It turns out that, like the $\mathrm{GH}$ spatial shift, the angular shift defined by Eq.(2b) is a quantum selection from multiple eigenstates of reflection angle under the $\mathrm{GH}$ effect.

\section{B. Numerical Simulations for Device Optical Performance}

In optical switching operations, in accordance with our previous work [11], once the angular shift happens in the GH effect, all the possible angles of the reflected mode are possibly no longer the same due to this angular shift of GH effect. Then, in terms of the feasible fabrication condition for this project on the SOI platform, the parameters of the WCM based TO-DOS are designed as that the thicknesses of the $\mathrm{SiO}_{2} \mathrm{BOX}$ and silicon film are 1.0 and $2.0 \mu \mathrm{m}$, respectively, the top cladding layer is a $1.0 \mu \mathrm{m}$ thick $\mathrm{SiO}_{2}$ film, the rib width and height have been optimized to be 4.0 and $0.5 \mu \mathrm{m}$, respectively. Then, at the wavelength $\lambda=1550 \mathrm{~nm}$, with Eqs. (1)-(3) we obtain the relationship between the GH spatial and angular shifts and the incident angle for TE-mode by simulated analysis as shown in Figure 2, where the left and right vertical axes stand for the GH spatial and angular shifts, respectively. Note that the spatial shift can reach the range from a few to 10 s of microns, while the maximum angular shift is lower than $0.015 \mathrm{rad}$. What needs to be clarified is that our currently feasible design and fabrication accuracies can only allow the incident within $45^{\circ} \pm 1^{\circ}$, which is in the range of $3.0-10.0 \mu \mathrm{m}$, the angular shift is $0.013 \mathrm{rad}$.

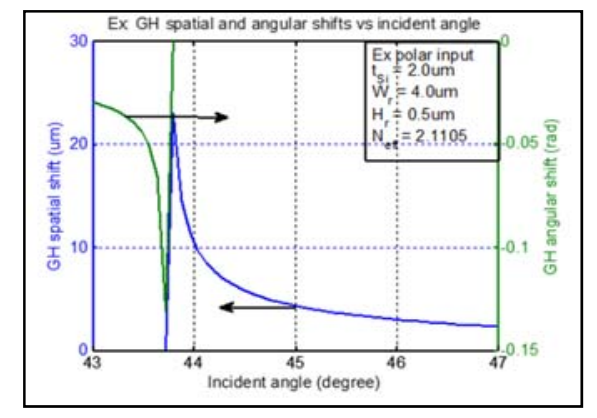

FIGURE II. NUMERICAL SIMULATIONS FOR THE DEPENDENCES OF THE GH SPATIAL AND ANGULAR SHIFTS ON THE INCIDENT ANGLE FOR TE-MODE

For the TO effect based refractive index modulation, there is a relationship between the refractive index modulation $\Delta n$ and the temperature increase $\Delta T$ as

$$
\Delta n=\frac{d n}{d T} \cdot \Delta T
$$

where $d n / d T$ stands for the thermo-optic coefficient of the modulated material. For the silicon material of SOI platform, $d n$ $/ d T=1.87 \times 10^{-4}\left(K^{-1}\right)$. FDTD software simulations show that the device shown in Figure 1 can work for TE-mode, so in the designs and experiments of a real device, only TE-mode performance is focused in the below research. In terms of the maintaining length of an MMI for a single-mode input signal, at wavelength $\lambda=1550 \mathrm{~nm}$ we set $l_{m m}=400 \mu \mathrm{m}$ and the input end width as $5.0 \mu \mathrm{m}$, then obtain the simulation results of the absolute displacements versus the temperature with respect to three incident angles as shown in Figure 3. Note that the initial GH shifts have differences for the different incident angles, but the GH shift jumps with the temperature are the same for all three incident angles. By comparing the results shown in Figure 3 with Figure 2, we conclude this attribute is owing to the event that in the dependence curve of spatial shift on the incident angle, the GH displacement jump is the slowly changes with a quasi-linear form rather than the sharp changing area around the critical angle when the incident angle is around $45^{\circ}$.

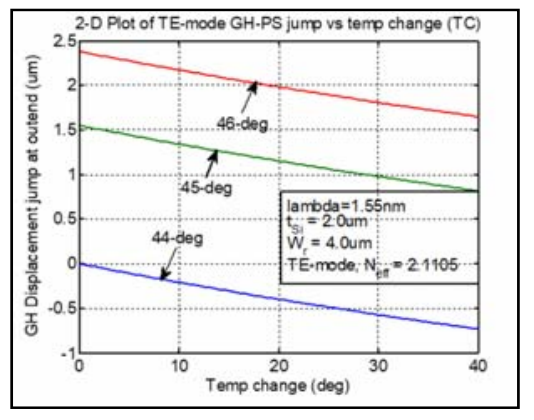

FIGURE III. SIMULATION RESULTS OF THE ABSOLUTE DISPLACEMENTS VERSUS THE TEMPERATURE WITH RESPECT TO THREE INCIDENT ANGLES AT TE-MODE FOR THE SOI WAVEGUIDE. 


\section{FDTD Simulations for Device Optical Performance}

With the structure for the numerical simulations shown in Figures 2 and 3, by setting the initial GH shift as $2.5 \mu \mathrm{m}$, and the angles of three output ports are at $45^{\circ}+1.0^{\circ}, 45^{\circ}, 45^{\circ}-1.0^{\circ}$. We also carry out the accurate simulations of optical loss performance with an FDTD software and then obtain the optical outputs at three ports as depicted in Table-1. Note that the optical on-chip losses at the upper, middle and lower ports are, respectively, $4.02,21.74$ and $28.54 \mathrm{~dB}$. Thus, one can conclude that the initial isolation between the upper port from the adjacent port, the middle one, is about $(21.74-4.02)=17.72 \mathrm{~dB}$. Similarly, we obtain the initial isolation between the lower port from the adjacent port, the middle one, is about (28.02 - 21.74) $=6.28 \mathrm{~dB}$. Thus, the output powers at the upper and middle ports can meet the requirement for the isolation between two adjacent ports, which can be used as two output ports of a switch.

TABLE I. FDTD SIMULATION RESULTS OF THE SOI-PIC 1X3 THERMO-OPTIC SWITCH AT OFF-STATE

\begin{tabular}{|c|c|c|c|}
\hline Item & \multicolumn{3}{|c|}{ Outputs at three ports } \\
\hline Number & Lower & Middle & Upper \\
\hline Data $(\mathrm{dB})$ & -28.02 & -21.74 & -4.02 \\
\hline
\end{tabular}

\section{EXPERIMENTS AND DISCUSSION}

To fabricate the experimental device samples of such a $1 \times 3$ TO DOS, the five devices having the same device structure as above simulated are designed to an array. Figure 4 show the photo image of a fabricated sample chip in which the corner mirrors, the heaters and soldering spots are labelled. Then, we measure the optical insertion losses (IL) of several 1x3 optical switch at the three output ports and the IL of the straight waveguide together with the $1 \times 3$ switch device, and then obtain the net structure-caused on-chip optical loss of $1 \times 3$ optical switch by subtracting the insertion losses of the straight waveguide from that of the switch device. Figure 5 depicts the measured results of the net on-chip optical insertion losses for several devices. Note that the expected output only has an average optical loss of $0.5-1.0 \mathrm{~dB}$ and an isolation of $14 \mathrm{~dB}$ from the middle port, which are consistent with the FDTD simulation values. In addition, the output powers among the five devices have uniform optical loss specifications.

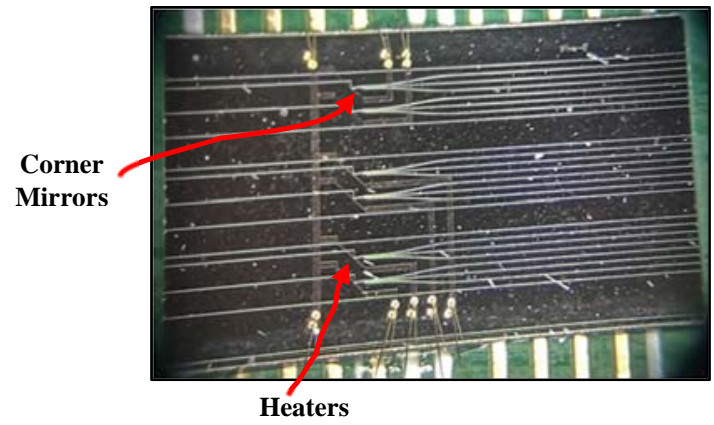

FIGURE IV. THE PHOTO-IMAGE OF THE FABRICATED DEVICE SAMPLE.

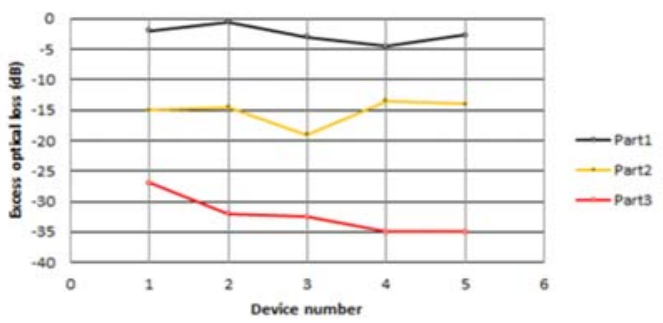

FIGURE V. THE MEASURED RESULTS OF OPTICAL ON-CHIP LOSSES AT THE THREE OUTPUT PORTS.

In the above experiments, the dynamic switching process of an input optical signal at the upper port is carried out under an electrical alternating signal as a heating driving source is shown in Figure 6 where the driving electrical signal has a frequency of $10 \mathrm{kHz}$, then the two switching times, the rise and fall times are $50 \mu$ s and $65 \mu$ s, respectively. Here what needs to be clarified is that the switching power is $150-200 \mathrm{~mW}$, which is higher than what an SOI TO switch should have. The direct reason for such a high switching power is this switch configuration has not reached the optimal eigenstate of quantum GH spatial shift at the current working state and incident angle, so it is the central content that needs to be improved in this work in future. In addition, as analyzed above, due to the limitation of fabrication condition, in the configuration of the $1 \times 3$ optical switch in this work, the GH angular shift has not been exploited yet, so the optical quantum effect of GH effect has not been developed out. Accordingly, the advancements of digital thermo-optic switching functions could not fully be reached with the current configuration.

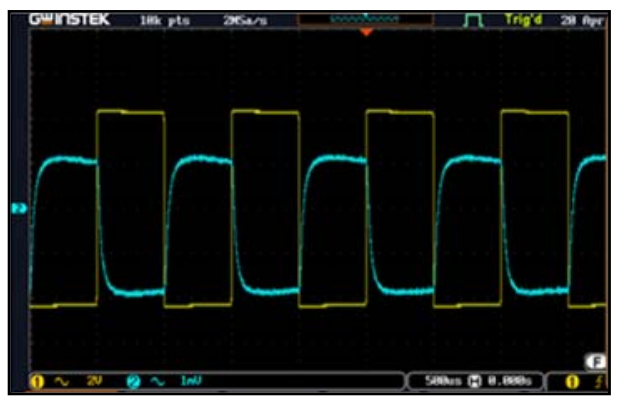

FIGURE VI. THE DYNAMIC SWITCHING PROCESS, WHERE AS LABELLED BY THE INSETS WHERE THE ALTERNATING ELECTRIC DRIVING SIGNAL HAS A 10K FREQUENCY AND 5V AMPLITUDE

\section{CONCLUSIONS}

As a conclusion, based on the quantum output selections of switching operations, the DOS regime in this work still has a large room to be improved in the device performance such as the switching power, the isolation, the switching power and the switching velocity with the further optimization of the device architecture albeit the measured values of device performance are not impressive. The comparison with the conventional SOIwaveguide TO switch has apparently presented the advancements in optical performance albeit the configuration studied in this work is not optimized. Thus, as the micro-size 
switch units, it will have the substantial applications in the undergoing large-scale matrix TO switches.

\section{ACKNOWLEDGMENT}

National Science Foundation of Jilin Provincial Science and Technology/China (20180101223JC, 20160101263JC); Natural Science Foundation of China (Young Grant: 11604025); The Innovation Fund of Changchun University of Science and Technology/China; The Talent Development Fund of HR Department of Jilin Province.

The authors would like to thank the involved post-graduates in the same group, Yue Han, Zhuo Chen, Bin Wang, and Ting $\mathrm{Yu}$ for their helps to this project in the chip processes of the experimental samples.

\section{REFERENCES}

[1] K. Chrostowski and M. Hochberg, "Silicon Photonics: Disruptive and Ready for Prime Time," in Silicon Photonics: Fueling the Next Information Revolution, D.Innis and R. Rubenstein 1st Ed, Part-1, Cambridge University Press, Cambridge, 2015.

[2] J.S. Orcutt, B. Moss, C. Sun, J. Leu, M. Georgas, J. Shainline E. Zgraggen, H. Li, J. Sun, M. Weaver, S. Uroševic, M. Popović, R. J. Ram, and V. Stojanović, "Open foundry platform for high-performance electronicphotonic integration," Optics Express, vol. 20, no. 11, pp. 12222-12232, 2012.

[3] D. G. Sun, Z. Hu, S. Majid, R. Vandusen, Q. Zheng, I. Hasan, N. G. Tarr, S. Bidnyk, and T. J. Hall, "Limitation factor analysis for silicon-oninsulator waveguide Mach-Zehnder interference based electro-optic switches,” IEEE/OSA Journal of Lightwave Technology, vol. 29, no. 17, pp. 2592-2600, 2011.

[4] S. Rumley et al., "Silicon photonics for extra-scale systems," IEEE/OSA Journal of Lightwave Technology, vol. 33, no. 3, pp. 547-562, 2015.

[5] M. R. Watts, J. Sun, C. DeRose, D. C. Trotter, R. W. Young, and G. N. Nielson, "Adiabatic thermo-optic Mach-Zehnder switch," Optics Letters, vol. 38, no. 5, pp. 733-735, 2013.

[6] L. Yang, Y. H. Xia, F. F. Zhang, Q. S. Chen, J. F. Ding, P. Zhou, and L. Zhang, "Reconfigurable nonblocking 4-port silicon thermo-optic optical router based on Mach-Zehnder optical switch," Optics Letters, vol. 40, no. 7, pp. 1402-1405, 2015.

[7] Y. Shoji, K. Kintaka, S. Suda, and H. Ishikawa, "Low crosstalk $2 \times 2$ thermo-optic switch with silicon wire waveguides," Optics Express, vol. 18, no. 9, pp. 9071-9075, 2010.

[8] R. Aguinaldo, A. Forencich, C. DeRose, A. Lentine, D. C. Trutter, Y. Fainman, G. Porter, G. Papen, and S. Y. Mookherjer, "Wideband siliconphotonic thermo-optic switch in a wavelength division multiplexed ring network," Optics Express, vol. 22, no. 7, pp. 8205-8218, 2014.

[9] R. Krahenbuhl, M. M. Howerton, J. Dubinger, and A. S. Greenblatt, "Performance and modeling of advanced Ti: LiNbO3 digital optical switches," IEEE/OSA Journal of Lightwave Technology, vol. 20, no. 1, pp. 92-99, 2002.

[10] D. G. Sun, "A proposal for digital electro-optic switches with free-carrier dispersion effect and Goos-Hanchen shift in silicon-on-insulator waveguide corner mirror," Journal of Applied Physics, vol. 114, no. 10, p. 104502, 2013.

[11] D. G. Sun, "Manipulation of the coherent spatial and angular shifts of Goos-Hänchen effect to realize the digital optical switch in silicon-oninsulator waveguide corner," Journal of Applied Physics, vol. 120, no. 18, p. 183101, 2016. 